\title{
Renal Involvement in Mediterranean Spotted Fever: Clinical and Histopathological Data
}

\author{
Ivan Baltadzhiev ${ }^{\mathrm{a}}$ Zaprian Zaprianov ${ }^{\mathrm{b}} \quad$ Atanas Baltadjiev $^{\mathrm{c}}$ \\ aDepartment of Infectious Diseases, Parasitology and Tropical Medicine, Faculty of Medicine, Medical University

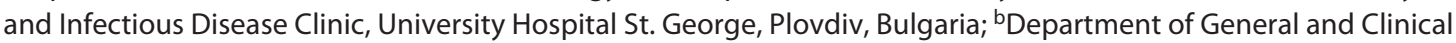 \\ Pathology, Faculty of Medicine, Medical University and General and Clinical Pathology Clinic, University Hospital \\ St. George, Plovdiv, Bulgaria; 'Department of Anatomy, Histology and Embryology, Faculty of Medicine, Medical \\ University, Plovdiv, Bulgaria
}

\section{Highlights of the Study}

- The purpose of this study was to investigate the kidney involvement and to detect renal histopathologic lesions among subjects with Mediterranean spotted fever.

- The results indicate that the renal damage consists of rickettsial vasculitis with disseminated perivascular lymphohistiocyte infiltrates.

\section{Keywords}

Mediterranean spotted fever · Renal complications ·

Histopathology

\begin{abstract}
Objective: Mediterranean spotted fever (MSF) is a tick-borne rickettsial infection endemic to the Mediterranean coastline countries. As a result of growing tourism, imported cases have been registered in many nonendemic countries and regions. We present clinical laboratory parameters and histopathological data on renal impairment in patients with MSF. The study meets our goal of identifying kidney involvement and detecting renal damage in people with MSF. Subjects and Methods: Three hundred fifty patients with MSF with a diagnosis confirmed by immunofluorescence analysis were tested for serum urea, creatinine, and albumin. Fifty-five patients with malignant form of MSF were divided into 2
\end{abstract}

groups: 19 fatalities and 36 survivors. The percentage of patients with acute renal failure (ARF) was compared in both groups. Results: Subjects with elevated urea and creatinine levels increased from 5.21 to $3.47 \%$ in mild to 48.78 and $29.26 \%$ in severe MSF, respectively. Loss of serum albumin also increased from mild to severe MSF. Renal impairment comprised $60 \%$ of the cohort of 55 patients with malignant MSF: $89.4 \%$ in the group of deaths and almost twice less in the survivors. ARF developed in $84.2 \%$ of fatal cases and was $>2$ times less in survivors. Postmortem light microscopy of renal samples of 9 fatal cases revealed perivascular mononuclear inflammatory infiltrates, vasculitis with fibrinoid necrosis, acute tubular necrosis, interstitial edema, hemorrhage, and thrombosis. Conclusion: Renal pathology associated with MSF rickettsial infection consists of systemic small vessel vasculitis and vascular injury, leading to ARF in the most severe cases.

(c) 2021 The Author(s)

Published by S. Karger AG, Basel karger@karger.com www.karger.com/mpp

Karger $\stackrel{\text { ' }}{5}$

BOPEN ACCESS
(C) 2021 The Author(s)

Published by S. Karger AG, Basel

This is an Open Access article licensed under the Creative Commons Attribution-NonCommercial-4.0 International License (CC BY-NC) (http://www.karger.com/Services/OpenAccessLicense), applicable to the online version of the article only. Usage and distribution for commercial purposes requires written permission.
Correspondence to:

Ivan Baltadzhiev, ivan_balt@yahoo.com 


\section{Introduction}

Mediterranean spotted fever (MSF) caused by Rickettsia conorii ( $R$. conorii) is a tick-borne infection endemic to the countries along the Mediterranean Sea coast, Southern Europe, North Africa, and India. However, as a result of growing tourism, imported cases have been reported in many nonendemic countries and regions $[1,2]$.

$R$. conorii is a small Gram-negative nonmotile, often very pleomorphic bacteria. It is a strictly intracellular pathogen, belonging to the spotted fever group (SFG) of genus Rickettsia of the family Rickettsiaceae. The life cycle of SFG rickettsiae involves insect vector and mammal reservoirs. $R$. conorii is transmitted to humans by the brown dog tick Rhipicephalus sanguineus, assumed to be both the vector and reservoir of infection. Humans are accidental hosts. Domestic and stray dogs play significant roles in the epidemiological chain as intermediaries between ticks and humans.

Complete sequencing of the $R$. conorii genome has established that the $R$. conorii conorii strain ATCC VR-613/ Malish 7 is the cause of MSF in the Mediterranean countries and in areas surrounding the Black Sea, including Bulgaria $[1,3,4]$. Thus, by molecular methods, it was found that the typical strain for MSF in the endemic regions in Bulgaria is caused by $R$. conorii conorii and is a separate nosological unit.

The first cases of MSF occurring as a small outbreak in the Plovdiv region of Bulgaria were described in 1948. In early 1993, MSF re-emerged and spread to new geographical areas of the country, with predominance in urban residents. The new wave showed more severe clinical forms, complications, and deaths. Although slowing down, it continues even now [5].

The main clinical signs and symptoms of MSF typical forms include a skin eschar (tache noire) at the site of tick bite, toxic-infectious syndrome (TIS; comprising all or some of the following flu-like symptoms: fever, chills, headache, arthralgia, myalgia, malaise, adynamia, dizziness, anorexia, nausea, vomiting, etc.), and maculopapular rash on the body and extremities, not sparing the palms and feet $[2,6]$. As with other infectious diseases, atypical forms have also been reported [7, 8]. MSF has usually a mild or moderate course and has long been considered a benign disease; however, severe forms including major neurological manifestations and multiorgan involvement may occur [9]. Actually, the so-called malignant forms of MSF with involvement of vital organ systems and often lethal outcomes have been observed [1012]. A hallmark of MSF pathology is the rickettsial vasculitis. Pathogenic rickettsiae infect and replicate in the vascular endothelial cells lining capillaries, arterioles, and venules. Their tropism toward microvascular endothelium leads to endothelial cell activation, disseminated inflammation, and altered vascular functions, consisting in compromised vascular integrity, increased permeability, and loss of barrier role $[13,14]$. Endothelial dysfunction during a rickettsial infection, termed rickettsial vasculitis, manifests as a variety of organ system impairment and complications. The aim of this study was to examine renal involvement and to detect histopathological lesions in the renal tissue of patients with MSF in order to assess the impact of acute kidney injury (AKI) on the course of the disease.

\section{Patients and Methods}

\section{Patients}

Our study data are based on the monitoring of 350 inpatients (169 men and 181 women, age: $15-78$ years) in the Infectious Diseases Clinic at the St. George University Hospital, the only medical facility serving and treating patients with MSF in Plovdiv and its suburbs, the largest MSF-endemic region in Bulgaria with approximately 700,000 inhabitants. The patients enrolled in the study were based on the following inclusion criteria: 1) development of febrile illness with flu-like symptoms 3-4 days prior to the onset of a typical maculopapular rash, 2) pathognomonic eschar (tache noire) or other presumed port of entry-conjunctiva, skin lesions, etc., 3) 4-fold increase in $R$. conorii antibody titer in repeated serum samples or appropriately elevated IgG and/or IgM titer, and 4) no anti-rickettsial antibiotic treatment was performed prior to hospitalization. Exclusion criteria included patients with coexisting acute or exacerbated chronic medical conditions, including any type of kidney abnormalities or disease.

We studied the renal involvement of 350 patients with MSF, divided into 3 groups: with mild (115), moderate (153), and severe (82), the last including 27 severe and 55 malignant form of the disease. All patients were tested for urea, creatinine, and albumin in the blood serum. The percentage of patients with elevated blood urea nitrogen (BUN) and creatinine levels was determined, as well as the percentage of patients with decreased serum albumin. The maximum deviation of these laboratory indicators from the norm is shown in Table 1. Fifty-five patients ( 30 men and 25 women) with a malignant form of MSF were divided into 2 groups: group I consists of 19 deaths with a mean age of $59.55 \pm 4.09$ years and group II consists of 36 survivors with a mean age of $47.22 \pm 5.81$ years (mean \pm SEM), $p>0.05$.

This study was approved by the Ethics Committee of Medical University of Plovdiv. An informed consent for the autopsy was signed by the relatives of the deceased patients.

Severity of MSF

Criteria determining the severity of MSF have been detailed in our previous study [15]. 
Table 1. Changes in urea, creatinine, and albumin levels in the blood serum of patients with MSF

\begin{tabular}{|c|c|c|c|c|c|c|c|}
\hline & $\begin{array}{l}\text { Total } \\
(n=350)\end{array}$ & $\begin{array}{l}\operatorname{mild}^{1} \\
(n=115)\end{array}$ & $\begin{array}{l}\text { moderate }^{2} \\
(n=153)\end{array}$ & $\begin{array}{l}\text { severe }^{3} \\
(n=82)\end{array}$ & $\begin{array}{l}p \\
\text { value }^{1-2}\end{array}$ & $\begin{array}{l}p \\
\text { value }^{2-3}\end{array}$ & $\begin{array}{l}p \\
\text { value }^{3-4}\end{array}$ \\
\hline \multicolumn{8}{|c|}{ Normal ranges of urea in the blood serum (male 3.2-8.9; female $2.6-7.2$ ) $\mathrm{mmol} / \mathrm{L}$} \\
\hline Mean \pm SEM & $6.83 \pm 0.28$ & $4.71 \pm 0.19$ & $5.91 \pm 0.31$ & $11.51 \pm 0.86$ & & & \\
\hline Patients with values above upper limit, $n(\%)$ & $61(17.42)$ & $6(5.21)$ & $15(9.80)$ & 40 (48.78) & $<0.01$ & $<0.001$ & $<0.001$ \\
\hline Mean \pm SEM & $89.26 \pm 2.34$ & $71.46 \pm 2.14$ & $85.19 \pm 2.63$ & $122.23 \pm 6.98$ & & & \\
\hline Patients with values above upper limit, $n(\%)$ & $38(10.85)$ & $4(3.47)$ & $10(6.53)$ & $24(29.26)$ & $<0.001$ & $<0.001$ & $<0.001$ \\
\hline Maximum creatinine increase, $\mathrm{mmol} / \mathrm{L}$ & 378 & 160 & 308 & 378 & & & \\
\hline \multicolumn{8}{|l|}{ Normal ranges of albumin levels in the blood serum $35-55 \mathrm{~g} / \mathrm{L}$} \\
\hline Mean \pm SEM & $37.56 \pm 1.17$ & $41.38 \pm 0.46$ & $37.45 \pm 0.38$ & $33.86 \pm 0.62$ & & & \\
\hline $\begin{array}{l}\text { Patients with albumin below the lower normal limit, } \\
n(\%)\end{array}$ & $212(60.57)$ & $35(30.43)$ & $108(70.58)$ & $69(84.14)$ & $<0.0001$ & $<0.0001$ & $<0.0001$ \\
\hline
\end{tabular}

MSF, Mediterranean spotted fever. ${ }^{*}$ Mann-Whitney U rank-sum test. ${ }^{1}$ Mild form of MSF. ${ }^{2}$ Moderate form of MSF. ${ }^{3}$ Severe form of MSF.

\section{Mild Form}

Mild or absent TIS: fever $38-38.5^{\circ} \mathrm{C}$, no chills or headache, moderate myalgia/arthralgia; scarce maculopapular rash; no significant changes in the laboratory parameters - slight variations in peripheral white blood cell count; platelet count $\geq 120 \times 10^{9} / \mathrm{L}$; a negligible increase in aminotransferase ALT/AST activity might happen.

\section{Moderate Form}

Marked TIS: fever around $39^{\circ} \mathrm{C}$, chills, and moderate headache; scattered maculopapular rash; a normal or indicative of peribronchial changes in radiography; minor hepatomegaly; moderate leukocytosis/leukopenia with shift to the left; $2-3$-fold increase in serum ALT/AST activity; platelet count $\geq 100$ but $<140 \times 10^{9} / \mathrm{L}$; serum sodium at the lower reference range $(136 \mathrm{mmol} / \mathrm{L})$; serum albumin at the lower reference range- $35 \mathrm{~g} / \mathrm{L} ; \mathrm{BUN}$ and creatinine within the reference ranges or mildly elevated.

\section{Severe Form}

Severe TIS: fever above $39^{\circ} \mathrm{C}$, chills, severe headache, and vomiting; abundant rash with hemorrhagic components; clinically and laboratory manifested damage to one organ in the human body (lung, liver, kidney, myocardium, etc.); platelet count $>50<120$ $\times 109 / \mathrm{L}$; serum sodium $\leq 136 \mathrm{mmol} / \mathrm{L} ; \mathrm{Ca}<2.12 \mathrm{mmol} / \mathrm{L} ; \mathrm{ALT} /$ AST activity increased $\geq 4-5$ times above normal; serum BUN and creatinine levels above reference limits.

\section{Malignant Form}

Very severe TIS: fever $\geq 40^{\circ} \mathrm{C}$, chills, severe headache, nausea, and repeated vomiting; typhoid mental state, stupor, or coma; abundant hemorrhagic rash; clinically and laboratory apparent lesions of $>1$ organ (pneumonitis, jaundice, renal failure, gastrointestinal hemorrhage, myocarditis, and CNS damage); platelet count $\leq 50$ or $<100 \times 10^{9} / \mathrm{L}$; serum sodium $<136 \mathrm{mmol} / \mathrm{L}$; ALT/ AST activity increase $>5$-fold over normal, hypocalcemia, hypoalbuminemia, and hypoxemia.

Renal Involvement in Mediterranean Spotted Fever

\section{Methods}

\section{Diagnosis of MSF}

The disease was diagnosed on the basis of epidemiological and clinical criteria and etiologically confirmed by a positive antibody response to a specific $R$. conorii antigen, measured by indirect immunofluorescent assay. Indirect immunofluorescent assay was performed in the Reference Laboratory for Rickettsiosis at the Military Medical Academy - Sofia (Rickettsia conori-Spot IF, BioMerieux, Marcy L'Etoile, France). The diagnosis of $R$. conorii infection is confirmed when acute serum contains IgM antibodies at a titer $\geq 64$ and/or IgG titer $\geq 128$ or when there is a 4 -fold increase in the titer between acute and convalescent sera. The treatment is based on antibiotic (doxycycline or macrolides) and resuscitation measures, including hemodialysis where appropriate.

\section{Histopathological Study}

To determine the nature of kidney damages in patients with fatal MSF, we examined renal tissue from 9 individuals ( 3 men and 6 women, aged $45-78$ years) who died of the malignant form of the disease. We performed a postmortem histopathological examination, including a complete light microscopic examination of the samples from the liver, lung, kidneys, stomach, pancreas, and brain. Samples were fixed in $4 \%$ neutral buffered solution of formaldehyde, embedded in paraffin, sectioned at 5- $\mu \mathrm{m}$ thicknesses, and processed by staining with hematoxylin/eosin to assess histopathology. Autopsies of the deceased patients were performed with informed consent signed by their relatives.

\section{Statistical Analysis}

Statistical analysis was performed using software package SPSS 15.0.1. Due to the lack of Gaussian distribution of the variables, the differences between the means were assessed for statistical significance using the nonparametric Mann-Whitney U test to compare the means of 2 groups, and the nonparametric Kruskal-Wallis ANOVA test - of $>2$ groups. Results were presented as mean \pm 
Table 2. Comparative assessment of renal involvement in fatalities (group I) and survivors (group II) in malignant forms of MSF

\begin{tabular}{|c|c|c|c|c|c|c|}
\hline & \multirow{2}{*}{$\begin{array}{l}\text { MSF total cases } \\
(n=55) \\
n(\%)\end{array}$} & \multirow{2}{*}{$\begin{array}{l}\text { MSF lethal cases } \\
(n=19) \\
\text { Group I } \\
n(\%)\end{array}$} & \multirow{2}{*}{$\begin{array}{l}\text { MSF survivors } \\
(n=36) \\
\text { Group II } \\
n(\%)\end{array}$} & \multicolumn{3}{|c|}{ Fisher's exact test } \\
\hline & & & & $p$ value & OR 95\% & $95 \% \mathrm{CI}$ \\
\hline Renal involvement & $33(60.00)$ & & & & & \\
\hline Very high urea and creatinine levels* & $33(60.00)$ & $17(89.47)$ & $16(44.44)$ & 0.001 & 10.025 & $2.13-52.95$ \\
\hline Acute renal failure & $30(54.54)$ & $16(84.21)$ & $14(38.88)$ & 0.001 & 8.381 & $2.05-34.11$ \\
\hline
\end{tabular}

OR, odds ratio; CI, confidence interval; MSF, Mediterranean spotted fever. * At least 3-4 times above the upper limit.

SEM. Fisher's exact test was used to compare the number of dead and surviving patients with malignant MSF. Two-tailed $p<0.05$ was considered statistically significant.

\section{Results}

It can be seen that the mean levels of both azotemic substances increase from mild to severe MSF and the differences are significant with a maximum increase several times higher than normal (Table 1). The percentage of patients with elevated levels of these 2 parameters, increasing from mild to severe forms of the disease is also indicative. For example, the percentage of patients with renal impairment ranged from $5.21 \%$ in mild to $48.78 \%$ in severe disease, and from 3.47 to $29.26 \%$ in the assessment of urea and creatinine levels, respectively. Both laboratory parameters reflect the increasing renal involvement in an increasing number of patients along with the severity of MSF. Changes in the level of serum albumin were observed in patients with various forms of severity. The loss of serum albumin, growing from mild to severe MSF is noteworthy (Table 1).

We examined a cohort of 55 subjects with the malignant form of MSF - 19 deaths and 36 survivors. The groups were compared in terms of both laboratory criteria - high serum urea and creatinine levels and one clinical criterion - the occurrence of acute renal failure (ARF). Renal function impairment covered $60 \%$ of the subjects $-89.47 \%$ in the group of deaths and almost twice less in the survivors. ARF was observed in $84.21 \%$ of the fatal cases and was more than twice as low in survivors (Table 2). We observed the following histopathological lesions in the renal tissue of 9 patients who died of malignant MSF: perivascular lymphohistiocytic inflammatory cell infiltrations were found in the kid- neys of all patients; histologic evidence of acute tubular necrosis with severe dystrophic to necrobiotic changes in the proximal tubule epithelium had 4 patients who developed ARF; extensive acute interstitial nephritis with interstitial edema was diagnosed in 3 patients, and focal interstitial nephritis with thrombotic vasculitis of the renal cortical and medullary microcirculation was observed in 2 of the deceased; symmetric cortical vasculitis with fibrinoid necrosis and microhemorrhages was also observed. Acute blood stasis and swelling in the glomerular mesangium were found in one of the autopsied patients. Some of the histopathological changes examined on kidney samples from deceased patients were taken on microphotographs, and shown in Figure 1.

As all deceased patients had a malignant form of MSF, in addition to the kidneys, many organs and systems were affected. For example, in 2 of the fatal cases, massive pulmonary thromboembolism occurred. Hemorrhagicnecrotic pancreatitis was demonstrated in 2 deaths. In 2 of the autopsied patients, acute ulcers were found along the small curve and in the bottom of the stomach with massive bleeding in the digestive tract. Pathological changes in the myocardium, brain, and other parenchymal organs have also been observed. Some of the patients survived from minutes to several hours after hospital admission.

\section{Discussion}

Renal involvement in MSF varies from mild or moderately elevated BUN retention to ARF. Rickettsia-induced renal damage leads to ARF by several mechanisms [16]. 


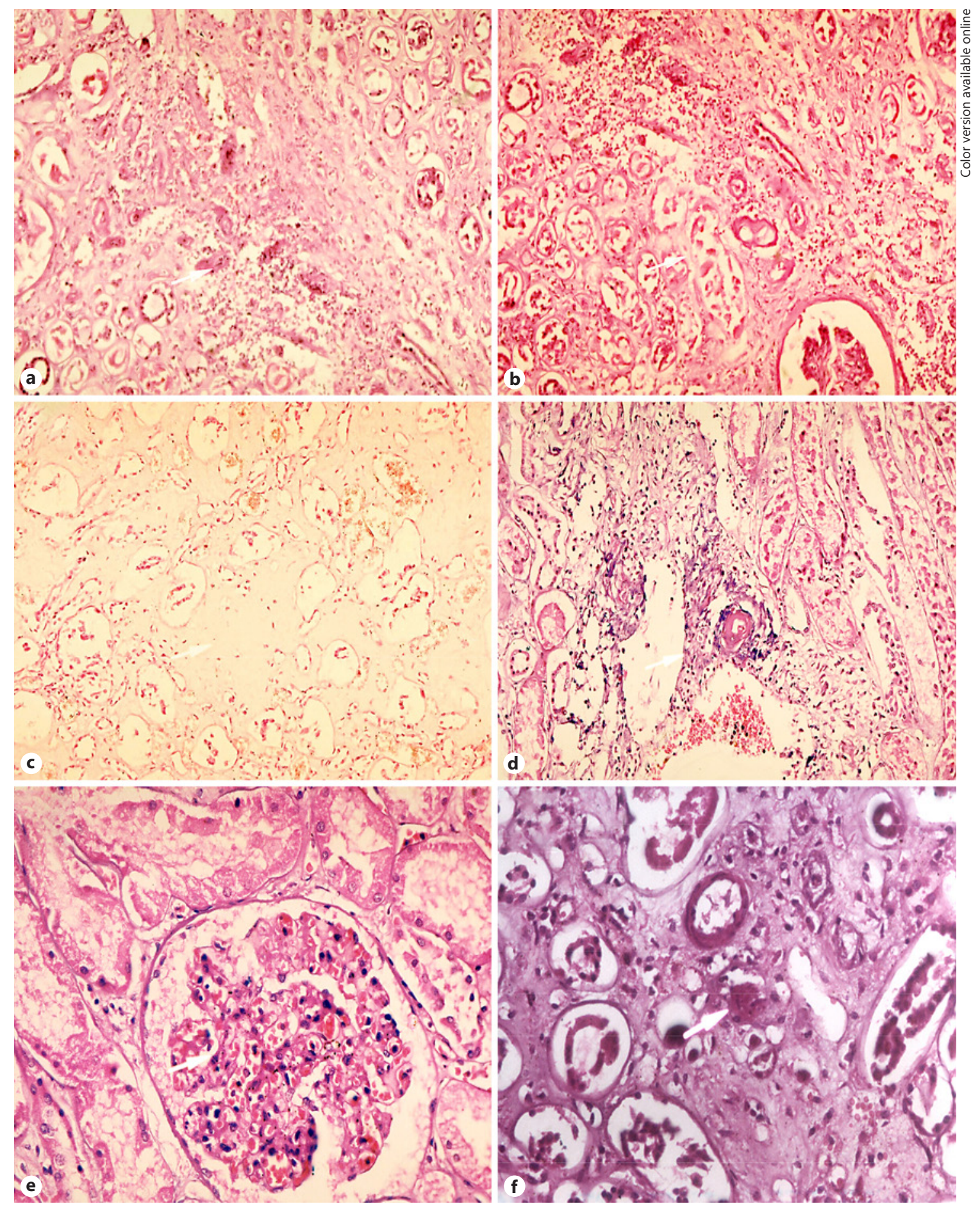

Fig. 1. Histopathological data on renal impairment in patients with MSF; cortical vasculitis with perivascular inflammatory mononuclear cell infiltration (a); hyaline microthrombi (arrowheads), interstitial edema and microhemorrhages, extensive interstitial inflammatory mononuclear cell infiltrate (b); insudation of blood plasma in the walls of the arterioles, interstitial edema, tubular necrosis of the proximal tubules (arrowheads) c Kidney medulla interstitial edema (arrowheads). Renal border zone (cortex-medulla)
- vasculitis with fibrinoid necrosis, necrosis of epithelial cells in the proximal tubules (arrowheads) (d); glomerulus - acute blood stasis; swelling in the mesangium (e); proteinuria in the Bowman's space (arrowhead); kidney - hyaline thrombus in an arteriole (arrowhead) (f). a-d, f - hematoxylin-eosin staining, original magnification $\times 200$; hematoxylin-eosin staining $(\mathbf{e})$, original magnification $\times 400$. MSF, Mediterranean spotted fever. 


\section{Prerenal Azotemia}

Based on the increased levels of serum urea and creatinine, renal function impairment may be suspected in approximately 17 and $10 \%$ of our MSF patients, respectively. Similar data have been reported by Crespo and colleagues [17] in Portugal; in a series of 250 patients with MSF, acute renal injury was found in $16 \%$ of the subjects, and this was the most common complication of the disease. We looked for markers of renal involvement in various forms of MSF, mild, moderate, and severe. In mild to moderate cases, the elevated urea and creatinine levels include a small percentage of patients. Moreover, the mean levels of these laboratory parameters do not exceed the reference ranges. The cause may be prerenal azotemia due to renal hypoperfusion from reduced renal blood flow of extra-renal origin (fever, dehydration, nausea, vomiting, and so on). In most cases, the increased levels of nitrogenous substances were predominantly in terms of BUN and with proper rehydration were usually overcome within $24 \mathrm{~h}$. According to Sahni et al. [18], AKI is usually a result of prerenal azotemia associated with hypovolemia and rarely acute tubular necrosis. However, severe forms of MSF with a high risk of morbidity and mortality are often associated with impaired renal function and AKI. The more intense and prolonged persistence of prerenal azotemia inherent in severe forms of the disease can cause severe impairment of renal perfusion and is often associated with ischemic tubular necrosis leading to ARF [19]. Nearly half and approximately onethird of our patients with severe MSF have elevated serum urea and creatinine levels, respectively. Hypovolemia early in the course of a rickettsial disease was reported to result in reversible prerenal azotemia; however, transient hypotension in the middle of the disease could produce acute tubular necrosis [16]. Systemic hypotension with decreased renal perfusion and glomerular filtration rate are the pathophysiologic mechanisms of higher incidence of ARF in fatal cases. At the heart of these mechanisms stands the systemic rickettsial vasculitis [13, 19-21].

\section{Rickettsial Vasculitis}

We observed deterioration in renal function in patients with malignant MSF and found that $60 \%$ of these patients had urea and creatinine levels 3-4 times higher than normal. Almost as many patients developed ARF. In fatal cases, $>89 \%$ of the deceased patients exceed the serum levels of these indicators, and in the survivors, the percentage is reduced by half. de Sousa et al. [20] reported fatal cases of $R$. conorii infection to be more likely to have elevated concentrations of serum urea and creati- nine at the time of admission; through multivariate analysis, these researchers identified ARF as an independent predictor associated with a fatal outcome from $R$. conorii infection [20].

Several researchers have postulated that the pathogenesis of rickettsial complications was a result of the widespread microvascular injury. Studies on the histopathology of various rickettsioses, especially the most severe one, Rocky Mountain spotted fever (RMSF), have shown the basic pathologic lesions consisting of focal or disseminated multiorgan vasculitis of small blood vessels; perivascular interstitial lymphohistiocytic infiltrates; increased vascular permeability with consequences of swelling, hypovolemia, hypotension, and hypoalbuminemia [16, 22]. Hypoalbuminemia due to leakage of plasma albumin into the perivascular space because of widespread vascular damage is common in patients with rickettsial disease, providing additional circumstantial evidence of increased capillary permeability [22]. Secondary microthrombosis and microinfarction of tissues irrigated by these vessels may be associated with acute tubular necrosis $[14,23]$.

There are relatively few histopathological examinations of the kidneys in patients with MSF. Shaked et al. [24] postulated that the principal histopathological lesion found in most severe cases of rickettsia-induced renal failure was multifocal perivascular interstitial nephritis. Later, Bellissima et al. [25] demonstrated interstitial nephritis and thromboangiitis, which required dialysis treatment in severe MSF. $R$. conorii was demonstrated previously in the vascular endothelium of the renal arterioles and capillaries, as well renal arteries and veins [26]. The sites of lesions and microorganisms showed strong topographical correlation, and rickettsiae were the apparent direct cause of other foci of vascular injury [26]. So, the actual mechanism of renal damage and complications concerns the direct effect of rickettsiae on kidney vascular endothelium.

Our histopathological findings are consistent with the mechanisms of systemic vasculitis, which reduce the effective renal blood flow due to increased vascular permeability. They are consistent with ischemic tubular necrosis and hypoalbuminemia because of plasma albumin leakage into the perivascular space by reason of widespread vascular injury. The described histopathological evidences in the kidneys of our patients who died of malignant MSF are similar to those found in the most severe SFG-rickettsiosis in America - RMSF. In fact, MSF has been stated to be a more severe disease than RMSF [1].

What is the impact of renal involvement on MSF mortality? Because all deceased patients had a malignant form of the disease, damage to many essential organs and sys- 
tems was the leading cause of death in the rickettsial infection. In fact, de Souza et al. [20] identified ARF as an independent predictor associated with a fatal outcome from MSF, and a recent report by us [11] is consistent with this suggestion. Interstitial nephritis cannot be conclusively demonstrated to contribute to ARF [16].

\section{Conclusion}

Renal pathology associated with MSF rickettsial infection consists of systemic vasculitis of small vessels and vascular damage leading to AKI and ARF in the most severe cases. This determines the need for its early diagnosis and appropriate treatment.

\section{Statement of Ethics}

Written informed consent was obtained from all patients for their participation in the study.

This study was approved by the Ethics Committee of Medical University of Plovdiv. An informed consent for the autopsy was signed by the relatives of the deceased patients.

\section{Conflict of Interest Statement}

The authors have no conflicts of interest to declare.

\section{Author Contributions}

Study design: I.B.; data collection: I.B. and Z.Z.; data interpretation: I.B., Z.Z., and A.B.; manuscript preparation: I.B., Z.Z., and A.B.; reference: I.B., Z.Z., and A.B.

\section{References}

1 Rovery C, Brouqui P, Raoult D. Questions on Mediterranean spotted fever a century after its discovery. Emerg Infect Dis. 2008;14(14): $1360-7$.

2 Parola P, Paddock CD, Socolovschi C, Labruna MB, Mediannikov O, Kernif T, et al. Update on tick-borne rickettsioses around the world: a geographic approach. Clin Microbiol Rev. 2014 Jan;27(1):166.

3 Fournier PE, Raoult D. Intraspecies diversity of Rickettsia conorii. J Infect Dis. 2009 Apr 1; 199(199):1097-9.

4 Blanda V, D'Agostino R, Giudice E, Randazzo K, La Russa F, Villari S, et al. New real-time PCRs to differentiate Rickettsia spp. and Rickettsia conorii. Molecules. 2020 Sep 27; 25(25):4431.

5 Baltadzhiev IG, Popivanova NI. Some epidemiological features of the Mediterranean spotted fever re-emerging in Bulgaria. Folia Med. 2012;54(1):36-43.

6 Faccini-Martínez ÁA, García-Álvarez L, Hidalgo M, Oteo JA. Syndromic classification of rickettsioses: an approach for clinical practice. Int J Infect Dis. 2014 Nov;28(28):126-39.

7 Baltadzhiev I, Popivanova N. Unusual maculopapular rash on the scalp of a patient with Mediterranean spotted fever. Med Princ Pract. 2019;28(28):291-3.

8 Fiore V, Mancini F, Ciervo A, Bagella P, Peruzzu F, Nunnari G, et al. Tache noire in a patient with acute $\mathrm{Q}$ fever. Med Princ Pract. 2018;27(27):92-4.

9 Brouqui P, Parola P, Fournier PE, Raoult D. Spotted fever rickettsioses in southern and eastern Europe. FEMS Immunol Med Microbiol. 2007 Feb;49(1):2-12.
10 Raoult D, Gallais H, Ottomani A, Resch JP, Tichadou D, De Micco P, et al. (Malignant form of Mediterranean boutonneuse fever. 6 cases). Presse Med. 1983;12(12):2375-8.

11 Baltadzhiev I, Popivanova N, Zaprianov Z. Malignant forms of Mediterranean spotted fever: risk factors for fatal outcomes. Braz J Infect Dis. 2016;20(5):511-2.

12 Demeester R, Claus M, Hildebrand M, Vlieghe E, Bottieau E. Diversity of life-threatening complications due to Mediterranean spotted fever in returning travelers. J Travel Med. 2010;17(17):100-4.

13 Walker DH, Mattern WD. Rickettsial vasculitis. Am Heart J. 1980 Dec;100(6 Pt 1):896906.

14 Vaphiades MS. Rickettsiae, rickettsial-like organisms, and the diseases they produce. In: Miller NR, Newman NJ, Biousse V, Kerrison JB, editors. Walsh \& Hoyt's clinical neuro ophthalmology. Philadelphia: Williams and Wilkins. p. 3050-52.

15 Baltadzhiev IG, Popivanova NI, Stoilova YM, Kevorkian AK. Mediterranean spotted feverclassification by disease course and criteria for determining the disease severity. Folia Med. 2012;54(4):53-61.

16 Walker DH, Mattern WD. Acute renal failure in rocky mountain spotted fever. Arch Intern Med. 1979;139:443-8.

17 Crespo P, Seixas D, Marques N, Oliveira J, da Cunha S, Meliço-Silvestre A. A Mediterranean spotted fever: case series of 24 years (1989-2012). Springerplus. 2015 Jun 17;4: 272.

18 Sahni A, Fang R, Sahni SK, Walker DH. Pathogenesis of rickettsial diseases: pathogenic and immune mechanisms of an endotheliotropic infection. Annu Rev Pathol. 2019 Jan 24;14(14):127-52.
19 Montasser DI, Zajjari Y, Alayoud A, Bahadi A, Aatif T, Hassani K, et al. (Acute renal failure as a complication of Mediterranean spotted fever). Nephrol Ther. 2011;7(4):245-7.

20 de Sousa R, França A, Nòbrega SD, Belo A, Amaro M, Abreu T, et al. Host- and microberelated risk factors for and pathophysiology of fatal Rickettsia conorii infection in Portuguese patients. J Infect Dis. 2008 Aug 15; 198(4):576-85.

21 Skhiri H, Zellama D, Aloui S, Bouraoui S, Frih MA, Achour A, et al. Acute renal insufficiency in boutonneuse mediterranean fever: description of three cases. Med Trop. 2004;64(1):5860.

22 Bradford WD, Croker BP, Tisher CC. Kidney lesions in rocky mountain spotted fever: a light-, immunofluorescence-, and electronmicroscopic study. Am J Pathol. 1979 Nov; 97(97):381-92.

23 Procop GW, Pritt B. Pathology of infectious diseases. John R Goldblum. E-Book, Elsevier, ExpertConsult com.

24 Shaked Y, Shpilberg O, Samra Y. Involvement of the kidneys in Mediterranean spotted fever and murine typhus. Q J Med. 1994;87(2):1037.

25 Bellissima P, Bonfante S, La Spina G, Turturici MA, Bellissima G, Tricoli D. (Complications of mediterranean spotted fever). Infez Med. 2001 Sep;9(3):158-62.

26 Walker DH, Gear JH. Correlation of the distribution of Rickettsia conorii, microscopic lesions, and clinical features in South African tick bite fever. Am J Trop Med Hyg. 1985; 34(34):361-71. 